\title{
Neonatal pemphigus: A case report at Dakar
}

\section{Khadim Diop, Boubacar Ahy Diatta, Niare Ndour, Saer Diadie, Aminata Deh, Coumba Ndiaye, Mamadou Sarr, Maodo Ndiaye, Moussa Diallo, Suzanne Oumou Niang}

Service de Dermatologie, Hôpital Aristide Ledantec, Dakar, Senegal

Corresponding author: Khadim Diop, MD, E-mail: bambadiop100391@gmail.com

\begin{abstract}
Neonatal pemphigus is an uncommon reported transient neonatal bullous dermatosis. We report the case of a male neonate who presented at birth with extensive post bullous erosions on the trunk. The mother was 31 years admitted to hospital 15 days postpartum for pemphigus vulgaris. At birth, the newborn presented with large diffuse post bullous erosions on the trunk and upper limbs. The diagnosis of neonatal pemphigus was based on the diagnosis of pemphigus vulgaris in the mother, the clinical course, the positivity of anti-cellular substance antibodies and the skin histology. The evolution was unremarkable with local care after 4 weeks. Besides its rarity, our observation is particular by the spontaneous unremarkable outcome and its clinical presentation leading to a differential diagnosis concern with other neonatal bullous dermatoses.
\end{abstract}

Key words: Pemphigus; Neonatal; Bullae; Dakar 


\section{Une observation de pemphigus néonatal à Dakar}

\section{Khadim Diop, Boubacar Ahy Diatta, Niare Ndour, Saer Diadie, Aminata Deh, Coumba Ndiaye, Mamadou Sarr, Maodo Ndiaye, Moussa Diallo, Suzanne Oumou Niang}

Service de Dermatologie, Hôpital Aristide Ledantec, Dakar, Senegal

Corresponding author: Khadim Diop, MD, E-mail: bambadiop100391@gmail.com

\section{RÉSUMÉ}

Le pemphigus néonatal est une dermatose bulleuse néonatale transitoire, rarement décrit. Nous rapportons le cas d'un nouveau-né de sexe masculin qui présentait à la naissance de larges érosions post bulleuses étendues sur le tronc. La mère était âgée de 31 ans, hospitalisée à 15 jours du post-partum pour un pemphigus vulgaire. Lexamen du nouveau-né retrouvait de larges érosions post bulleuses diffuses sur le tronc et les membres supérieurs. Le diagnostic de pemphigus néonatal était retenu devant le diagnostic de pemphigus vulgaire chez la mère, la présentation clinique, la positivité des anticorps anti-substance intercellulaire et l'histologie cutanée. L'évolution était favorable avec des soins locaux au bout de 4 semaines. Notre observation est particulière en plus de sa rareté par sa présentation clinique posant un problème de diagnostic différentiel avec les autres dermatoses bulleuses néonatales et l'évolution spontanément favorable.

Mots clés: Pemphigus; Néonatal; Bulles; Dakar

\section{INTRODUCTION}

Le pemphigus néonatal est une dermatose bulleuse intraépidermique transitoire, lié au passage transplacentaire d'autoanticorps anti substance inter-cellulaire [SIC] de type IgG maternels qui se fixent sur les antigénes desmogléine 3 des kératinocytes du nouveau-né chez une mère atteinte de pemphigus [1-3]. Il est rarement décrit dans la littérature médicale, environ une vingtaine de cas rapportés dans le monde depuis la première description en 1975 [4,5]. Il se manifeste à la naissance par des bulles flasques associées à de larges décollements épidermiques, posant un problème de diagnostic différentiel avec les autres dermatoses bulleuses néonatales. C'est une affection bénigne transitoire avec une régression des signes cliniques au bout de 3 semaines [6,7]. Nous rapportons une observation de pemphigus néonatal à Dakar.

\section{OBSERVATION}

Un nouveau-né de sexe masculin était reçu à la naissance au service de néonatologie de l'Hôpital Principal de Dakar pour de larges érosions post bulleuses étendues. Il était issu d'un accouchement par voie basse à domicile sans assistance médicale. La grossesse était mal suivie avec une consultation prénatale. La mère était âgée de 31 ans, 4G 4P, sans antécédents médicaux et gynéco-obstétricaux particuliers. Elle était hospitalisée à 15 jours du post-partum au service de Dermatologie de l'Hôpital Aristide Le Dantec pour des lésions bulleuses flasques étendues à $40 \%$ de la surface corporelle associées à des érosions aphtoïdes de la muqueuse buccale survenues depuis le sixième mois de la grossesse (24 semaines d'aménorrhée) (Fig.). Le diagnostic de pemphigus vulgaire était retenu chez la mère devant la présentation clinique, l'immunofluorescence indirecte avec la positivité des anticorps anti-substance intercellulaire à 16 fois la normale et l'histopathologie cutanée d'une bulle qui montrait un décollement bulleux intra-épidermique.

A l'admission, le nouveau-né était agité avec des pleurs incessants. La température était à $36,8^{\circ} \mathrm{C}$, le pouls à 120 battements/minute, la fréquence respiratoire à 28 cycles/minute, la glycémie capillaire à $0,9 \mathrm{~g} / \mathrm{dl}$ et le poids à 3100 grammes. Lexamen dermatologique notait de larges érosions post-bulleuses, avec un signe de Nikolski

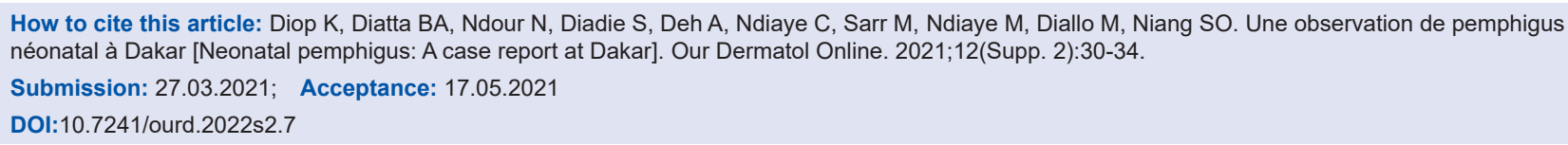


positif, étendues sur le tronc et les membres supérieurs sans atteinte des muqueuses (Fig. 2). Le reste de l'examen physique était normal. Limmunofluorescence indirecte notait une positivité des anticorps antisubstance intercellulaire à 8 fois la normale. La biopsie cutanée d'une bulle notait un décollement bulleux intra-épidermique. Limmunofluorescence directe et l'ELISA n'étaient pas réalisés car non disponibles. Le diagnostic de pemphigus néonatal était retenu devant le diagnostic de pemphigus vulgaire retenu chez la mère avec un début d'évolution des lésions au cours de la grossesse, la présentation clinique, la positivité des anticorps anti-substance intercellulaire et l'histopathologie cutanée. A l'hémogramme, les leucocytes étaient à $12300 / \mathrm{mm}^{3}$, le taux d'hémoglobine à $12,3 \mathrm{~g} / \mathrm{dl}$ et les plaquettes à $412000 / \mathrm{mm}^{3}$. La fonction rénale, l'ionogramme sanguin et les transaminases étaient normaux. Le traitement instauré était des soins locaux quotidiens avec attouchement à l'éosine aqueuse $2 \%$, une antibioprophylaxie à base de lincomycine injectable, une réhydratation et un allaitement artificiel. Lévolution était favorable marquée par une cicatrisation complète des lésions et une négativité des anticorps anti-substance intercellulaire au bout de 4 semaines, sans récidive notée après deux ans de recul (Fig. 3). La mère était traitée par une corticothérapie orale à base de Prednisone à $1 \mathrm{mg} / \mathrm{kg} / \mathrm{jour}$, des soins locaux quotidiens, avec une évolution favorable après un recul de deux mois (Fig. 4).

\section{DISCUSSION}

Nous avons rapporté un cas de pemphigus néonatal particulier en plus de sa rareté, par sa présentation clinique posant un problème de diagnostic différentiel

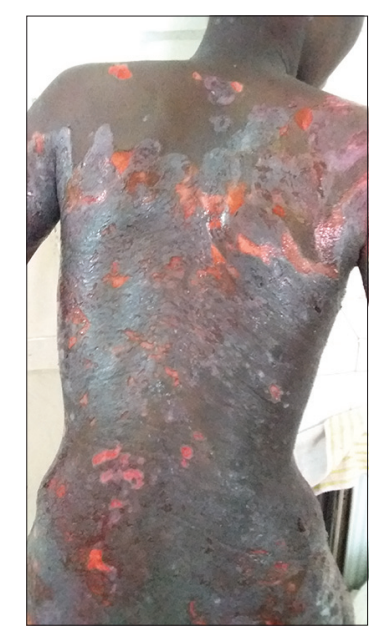

Figure 1: Erosions post bulleuses diffuses sur le tronc chez la mère avec les autres dermatoses bulleuses néonatales et son évolution spontanément favorable au bout de 4 semaines.

Le pemphigus néonatal est une dermatose bulleuse néonatale transitoire, caractérisé par la formation de bulles intra-épidermiques, induit par le transfert passif transplacentaire des anticorps antisubstances intercellulaires d'une mère atteinte de pemphigus vulgaire. En effet, les auto-anticorps anti-substances inter-cellulaires de type $\operatorname{IgG}$ maternels se fixent contre les protéines d'ancrage [Desmogléine 3] des kératinocytes des couches profondes de l'épiderme du nouveau-né et entraînent une dislocation des connections intercellulaires [5]. Le pemphigus néonatal est rarement décrit dans la littérature médicale, depuis la première description en 1975 par Rucco et al., depuis une trentaine de cas sont rapportés dans le monde [8]. Laspect clinique à la naissance est stéréotypé de simples vésiculobulles flasques à des atteintes érosives extensives, avec atteinte muqueuse possible [2]. Dans notre cas la présentation clinique était à type de bulles flasques associées à de larges érosions extensives. La présentation clinique faisait aussi évoquer les autres dermatoses bulleuses néonatales notamment une épidermolyse bulleuse héréditaire, une épidermolyse staphylococcique, une mastocytose cutanée et un incontinentia pigmenti. Le diagnostic de pemphigus vulgaire retenu chez la mère avec un début d'évolution lors de la grossesse, la positivité des anticorps anti-substance intercellulaire chez le nouveau-né et l'histopathologie cutanée ont permis de retenir le diagnostic de pemphigus néonatal.

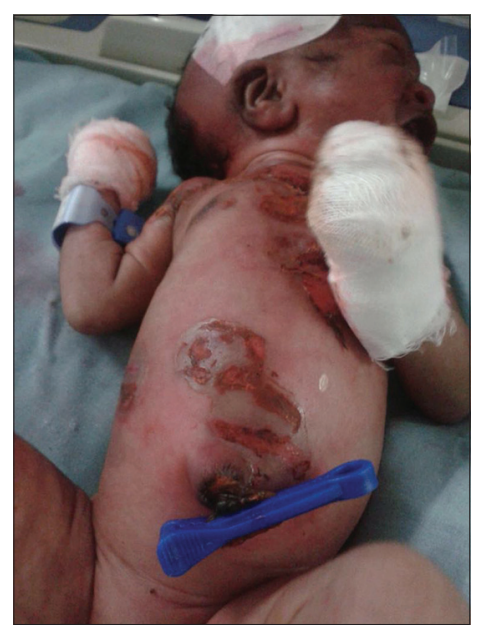

Figure 2: Bulles flasques avec Nikolski positif diffuses sur le tronc et les membres 


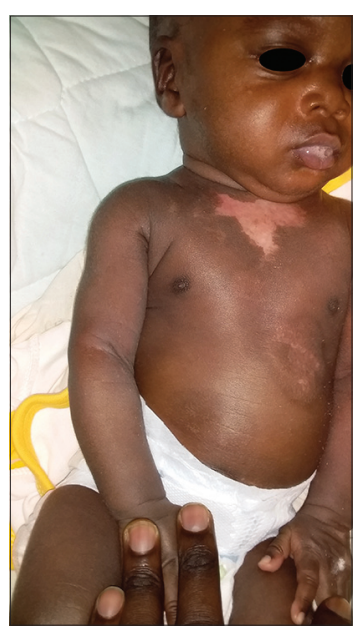

Figure 3: Cicatrisation des lésions après 4 semaines

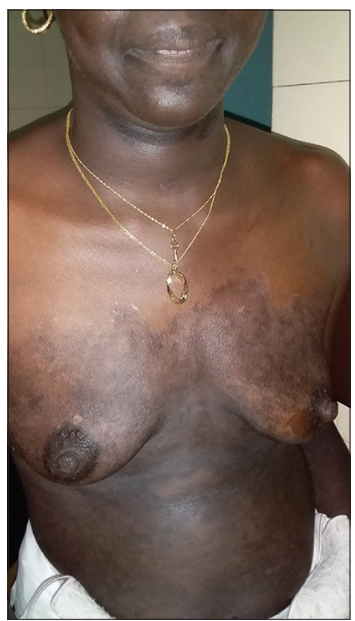

Figure 4: Cicatrisation des lésions chez la mère

Dans nos régions, les moyens diagnostics du pemphigus sont limités du fait de l'indisponibilité de l'immunofluorescence directe et de l'ELISA qui permettent une confirmation diagnostique [6]. L'immnufluorescence directe sur une peau péribulleuse objective des dépôts d'immunoglobulines $\mathrm{G}$ et/ou C3 au niveau des membranes cytoplasmiques des kératinocytes et donnant un aspect caractéristique en " mailles d'un filet”, qui est pathognomonique.

La survenue du pemphigus au cours de la grossesse est rarement rapportée, environ une cinquantaine de cas sont décrits dans la littérature médicale [6]. La maladie peut survenir à n'importe quel stade de la grossesse ou du post-partum. L'incidence d'un pemphigus néonatal au cours de la grossesse d'une mère atteinte de pemphigus vulgaire est de 30 à $45 \%$ [6]. Il n'y'a pas de corrélation établie entre le titre d'anticorps maternels de pemphigus vulgaire et la présentation de la maladie chez le nouveau-né [8-10]. Ceci est étayé par des cas de pemphigus néonatal de mères sans maladie active et des cas de nouveau-nés en bonne santé nés de mères atteintes d'une maladie hautement active [8,9]. La mort foetale peut survenir dans 5 à $12 \%$ des cas justifiant donc une surveillance fotale accrue $[11,12]$. D'autres complications à type de prématurité et d'avortement peuvent être observées [7].

Le pemphigus néonatal est une affection transitoire de bon pronostic. L'évolution des lésions est spontanément favorable au bout de 3 à 4 semaines avec les soins locaux, comme constatée dans notre cas $[6,8]$. Ceci résulte de la disparition spontanée des anticorps maternels IgG durant cette période. Aucun cas de récidive ou d'évolution vers un pemphigus à l'âge adulte n’a été rapporté à ce jour [2].

\section{Consent}

The examination of the patient was conducted according to the principles of the Declaration of Helsinki.

The authors certify that they have obtained all appropriate patient consent forms, in which the patients gave their consent for images and other clinical information to be included in the journal. The patients understand that their names and initials will not be published and due effort will be made to conceal their identity, but that anonymity cannot be guaranteed.

\section{RÉFÉRENCES}

1. Parlowsky T, Welzel J, Amagai M, Zillikens D, Wygold T. Neonatal pemphigus vulgaris: IgG4 autoantibodies to desmoglein 3 induce skin blisters in newborns. J Am Acad Dermatol 2003;48:623-5.

2. Panko J, Florell SR, Hadley J, Zone J, Leiferman K, Vanderhooft S. Neonatal pemphigus in an infant born to a mother with serologic evidence of both pemphigus vulgaris and gestational pemphigoid. J Am Acad Dermatol. 2009;60:1057-62.

3. Kader Ibrahim SB, BM Y, Umakanth S, Kanagasabai S. Pemphigus vulgaris in a pregnant woman and her neonate. BMJ Case Rep . 2012;2012:bcr0220125850.

4. Fenniche S, Benmously R, Marrak H, Dhaoui A, Ammar FB, Mokhtar I. Neonatal pemphigus vulgaris in an infant born to a mother with pemphigus vulgaris in remission. Pediatr Dermatol. 2006;23:124-7.

5. Masson P, Gaudy-Marqueste C, Es Sathi A, Tizeggaghine A, Touati K. Pemphigus vulgaire néonatal [Neonatal pemphigus vulgaris]. Arch Pediatr. 2001;8:1136-7.

6. Carvalho AA, Santos Neto DAD, Carvalho MADR, Eleutério SJP, Xavier AREO. Neonatal pemphigus in an infant born to a mother with pemphigus vulgaris: a case report. Rev Paul Pediatr. 2019;37:130-4.

7. Fainaru O, Mashiach R, Kupferminc M, Shenhav M, Pauzner D, Lessing JB. Pemphigus vulgaris in pregnancy: a case report and review of literature. Hum Reprod. 2000;15:1195-7.

8. Amer $\mathrm{YB}, \mathrm{Al}$ Ajroush W. Pemphigus vulgaris in a neonate. Ann Saudi Med. 2007;27:453-5.

9. Campo-Voegeli A, Muñiz F, Mascaró JM, García F, Casals M, 


\section{www.odermatol.com}

Arimany JL, et al. Neonatal pemphigus vulgaris with extensive mucocutaneous lesions from a mother with oral pemphigus vulgaris. Br J Dermatol. 2002;147:801-5.

10. Kalayciyan A, Engin B, Serdaroglu S, Mat C, Aydemir EH, Kotogyan A. A retrospective analysis of patients with pemphigus vulgaris associated with pregnancy. Br J Dermatol 2002;147:396-7.

11. Kardos M, Levine D, Gürcan HM, Ahmed RA. Pemphigus vulgaris in pregnancy: analysis of current data on the management and outcomes. Obstet Gynecol Surv. 2009;64:739-49.
12. Daneshpazhooh M, Chams-Davatchi C, Valikhani M, Aghabagheri A, Mortazavizadeh SM, Barzegari M, et al. Pemphigus and pregnancy: a 23-year experience. Indian J Dermatol Venereol Leprol. 2011;77:534.

Copyright by Khadim Diop, et al. This is an open-access article distributed under the terms of the Creative Commons Attribution License, which permits unrestricted use, distribution, and reproduction in any medium, provided the original author and source are credited.

Source of Support: Nil, Conflict of Interest: None declared. 